\title{
BMJ Open Cohort profile: InTraUterine sampling in early pregnancy (ITU), a prospective pregnancy cohort study in Finland: study design and baseline characteristics
}

\author{
Tuomas Kvist (D) , ${ }^{1}$ Sara Sammallahti, ${ }^{2}$ Marius Lahti-Pulkkinen, ${ }^{1}$ \\ Cristiana Cruceanu, ${ }^{3}$ Darina Czamara, ${ }^{3}$ Linda Dieckmann, ${ }^{3}$ Alina Tontsch, ${ }^{3}$ \\ Simone Röh, ${ }^{3}$ Monika Rex-Haffner, ${ }^{3}$ Eiina Wolford, ${ }^{1}$ Rebecca Reynolds, ${ }^{4}$ \\ Johan Eriksson, ${ }^{5,6}$ Sanna Suomalainen-König, ${ }^{7}$ Hannele Laivuori, ${ }^{8,9}$ \\ Eero Kajantie, ${ }^{10,11}$ Eija Lahdensuo, ${ }^{1}$ Elisabeth Binder, ${ }^{3}$ Katri Räikkönen ${ }^{1}$
}

To cite: Kvist T, Sammallahti S, Lahti-Pulkkinen M, et al. Cohort profile: InTraUterine sampling in early pregnancy (ITU), a prospective pregnancy cohort study in Finland: study design and baseline characteristics. BMJ Open 2022;12:e049231. doi:10.1136/ bmjopen-2021-049231

- Prepublication history for this paper is available online. To view these files, please visit the journal online (http://dx.doi. org/10.1136/bmjopen-2021049231).

Received 19 January 2021 Accepted 11 January 2022

Check for updates

(C) Author(s) (or their employer(s)) 2022. Re-use permitted under CC BY-NC. No commercial re-use. See rights and permissions. Published by BMJ.

For numbered affiliations see end of article.

Correspondence to

Dr Katri Räikkönen;

katri.raikkonen@helsinki.fi

\section{ABSTRACT}

Purpose The InTraUterine sampling in early pregnancy (ITU) is a prospective pregnancy cohort study. The overarching aim of ITU is to unravel genomic, epigenomic, transcriptomic, endocrine, inflammatory and metabolic maternal-placental-fetal mechanisms involved in the programming of health and disease after exposure to prenatal environmental adversity, such as maternal malnutrition, cardiometabolic disorders, infections, medical interventions, mental disorders and psychosocial stress. This paper describes the study protocol, design and baseline characteristics of the cohort.

Participants We included 944 pregnant Finnish women, their partners and children born alive between April 2012 and December 2017. The women were recruited through the national, voluntary trisomy 21 screening between $9^{+0}$ and $21^{+6}$ gestational weeks. Of the participating women, 543 were screen positive and underwent fetal chromosomal testing. Test result of these women suggested no fetal chromosomal abnormality. Further, we recruited 401 women who were screen negative and who did not undergo fetal chromosomal testing.

Findings to date We have collected chorionic villi and amniotic fluid from the screen-positive women; blood, urine, buccal swabs and diurnal salivary samples from all women; blood and buccal swabs from all partners; and placenta, cord blood and buccal swabs from all newborns for analyses of the genome, epigenome, transcriptome, and endocrine, inflammatory and metabolic markers. These data are coupled with comprehensive phenotypes, including questions on demographic characteristics, health and well-being of the women and their partners during pregnancy and of the women and their children at the child's age of 1.7 and 3 years. Data also come from patient records and nationwide registers covering health, lifestyle and medication data.

Future plans Multiple layers of ITU data allow integrative data analyses, which translate to biomarker identification and allow risk stratification and understanding of the biological mechanisms involved in prenatal programming of health and disease.

\section{Strengths and limitations of this study}

- This cohort is unique as it prospectively collected a wide range of biological tissue samples from the mother, her partner, the placenta and the fetus, which are embedded in the context of a rich set of comprehensive phenotypes that come from questionnaires, patient records and nationwide medical registers.

- InTraUterine sampling in early pregnancy (ITU) collected rare early-pregnancy chorionic villus and amniotic fluid samples, which allow studying biological mechanisms of programming during early pregnancy.

- ITU is ideally suited to study placental development as early-pregnancy chorionic villus samples can be matched to placenta biopsies sampled after delivery and be linked with other biomarkers and phenotypes.

- ITU cohort is relatively small $(n=944)$; nevertheless, multiple layers of data will allow integrative data analyses which will advance understanding of the biological mechanisms involved in prenatal programming of health and disease.

- Due to the design of the cohort, pregnant women who underwent fetal chromosomal testing in early pregnancy are over-represented (52\%).

\section{INTRODUCTION}

According to the Developmental Origins of Health and Disease (DOHaD) framework, fetal exposure to environmental adversities may alter the structure and functioning of rapidly developing organs and organ systems and thereby increase the child's future risk of adverse health outcomes. ${ }^{1}$ Even minor changes in the organ structure and functioning can become magnified over time and have implications for later health. To date, mounting empirical evidence lends support 
for the DOHaD framework. This evidence comes from studies which have shown that fetal exposure to environmental adversities, such as maternal malnutrition, gestational diabetes and hypertensive disorders, infections, medical interventions (eg, antenatal corticosteroid treatment), and mental disorders and psychosocial stress, increases the child's risk for a vast range of physical and mental disorders with the risk persisting throughout the lifespan. ${ }^{2-4}$

However, the biological maternal-placental-fetal mechanisms that underlie the programming effects of prenatal environmental adversities remain uncertain. The underlying mechanisms may include endocrine, inflammatory, metabolic and cardiovascular changes in the mother, the placenta and the fetus. ${ }^{5-7}$ These changes may be reflected in alterations in placental structure ${ }^{8}$ and functioning and include modifications in placental epigenomic and transcriptomic and fetal epigenomic profiles. ${ }^{9}$ Moreover, the programming effects of prenatal environmental adversities may be regulated by the fetal genome. ${ }^{9} 10$

Several longitudinal pregnancy cohort studies have obtained maternal and placental and/or fetal biosamples to assess the biological mechanisms that may link prenatal environmental adversities with child outcomes. These include the Avon Longitudinal Study of Parents and Children from the UK, ${ }^{11}$ the Generation R Study from the Netherlands, ${ }^{12}$ the Norwegian Mother, Father and Child Cohort Study ${ }^{13}$ and the Prediction and Prevention of Preeclampsia and Intrauterine Growth Restriction ${ }^{14}$ study from Finland. Further similar pregnancy cohort studies are described in the cohort profile of the Pregnancy and Childhood Epigenetics (PACE) ${ }^{15}$ the Early Growth Genetics (EGG) and the Early Genetics and Lifecourse Epidemiology (EAGLE) consortia. ${ }^{16}$

However, the existing studies have some important limitations. First, the range of tissue types and biomarkers in the prior pregnancy cohorts is limited, and very few has collected early-pregnancy chorionic villus or amniotic fluid samples in viable pregnancies and then followed up on the mothers and children. Second, most existing studies have examined only a few candidate biomarkers of interest, most often in maternal saliva or blood, such as cortisol, high-sensitivity C-reactive protein or glycoprotein acetyls. ${ }^{17}$ Third, the studies are also limited by studying epigenetic modifications, such as DNA methylation, in only one tissue type, most often in the placenta or fetal cord blood collected at birth. ${ }^{9}$ Finally, most studies have examined gene expression of candidate genes and in just one tissue type, most often in the delivery placenta. ${ }^{18-20}$ We are not aware of previous studies that would have examined both epigenomic and transcriptomic modifications in parallel in one tissue type, such as chorionic villi, or that would have studied these modifications longitudinally, such as in chorionic villi in early pregnancy and in matching delivery placentas.

The InTraUterine sampling in early pregnancy (ITU) study aims to overcome some of the key limitations of the existing pregnancy cohort studies. ITU study is, to our knowledge, the first study to couple genomic, epigenomic, transcriptomic and endocrine, inflammatory and metabolic data measured from unique maternal, placental and fetal tissue samples with a rich set of phenotypes on prenatal environmental adversities, demographic characteristics, lifestyle and health and disease of the mothers, their partners and children in a follow-up from the child's birth until the age of 3 years. With these data, we can address the overarching objective of the ITU study, which is to unravel the biological maternal-placental-fetal mechanisms that may be involved in prenatal programming of health and disease after exposure to prenatal environmental adversities. This cohort profile describes the design and provides an overview of multiple layers of data collected in ITU, reports results from a comparison between the ITU sample and a population-based sample comprising data from all pregnancies and births in Finland between 2006 and 2017 and describes ITU baseline characteristics

\section{COHORT DESCRIPTION}

\section{Study design and procedure}

The ITU study is a prospective pregnancy cohort study which includes 944 women residing in the area of the Helsinki and Uusimaa Hospital District, Finland, who gave birth to a live child between April 2012 and December 2017. Eligibility criteria included singleton pregnancy, no prenatal diagnosis of fetal chromosomal abnormality, maternal age $\geq 18$ years and sufficient Finnish language ability to ensure informed consent. Originally, 955 women met these criteria and consented to participate, but after the exclusion of participants whose pregnancy did not result in a live birth $(n=8)$ or whose child died within the first week of life $(n=1)$, and those who moved abroad before childbirth and whose child was thus not given a Finnish personal identification number $(\mathrm{n}=2)$, the final cohort included 944 mothers and their children and 480 maternal partners. Figure 1 shows the participant flow.

To elucidate the recruitment protocol, we first provide background information about prenatal screening in Finland. The national voluntary prenatal screening programme for trisomy 21 is available for all pregnant women in Finland free of charge: $~ 95 \%$ of pregnant women participate in this screening. ${ }^{21}{ }^{22}$ The screening programme includes a combination of serum screening (Pregnancy Associated Plasma Protein-A(PAPP-A) and free $\beta$-subunit of human chorionic gonadotropin ( $\beta$-hCG) at $9^{+0}-11^{+6}$ weeks of gestation; or a-fetoprotein, oestriol, $\beta$-hCG and inhibin-A at $15^{+0}-16^{+6}$ weeks of gestation) and ultrasound examinations (including a nuchal translucency scan at $10^{+0}-13^{+6}$ weeks of gestation, and a detailed examination of fetal structures at $18^{+0}-21^{+6}$ weeks of gestation). Approximately $5 \%$ of women have a positive screening result (ie, an estimated risk of fetal trisomy 21 $>1: 250$, based on serum and ultrasound screening, age, maternal height and weight and history), and are then 
Chromosomal testing arm mothers who underwent CVS, AC or NIPT

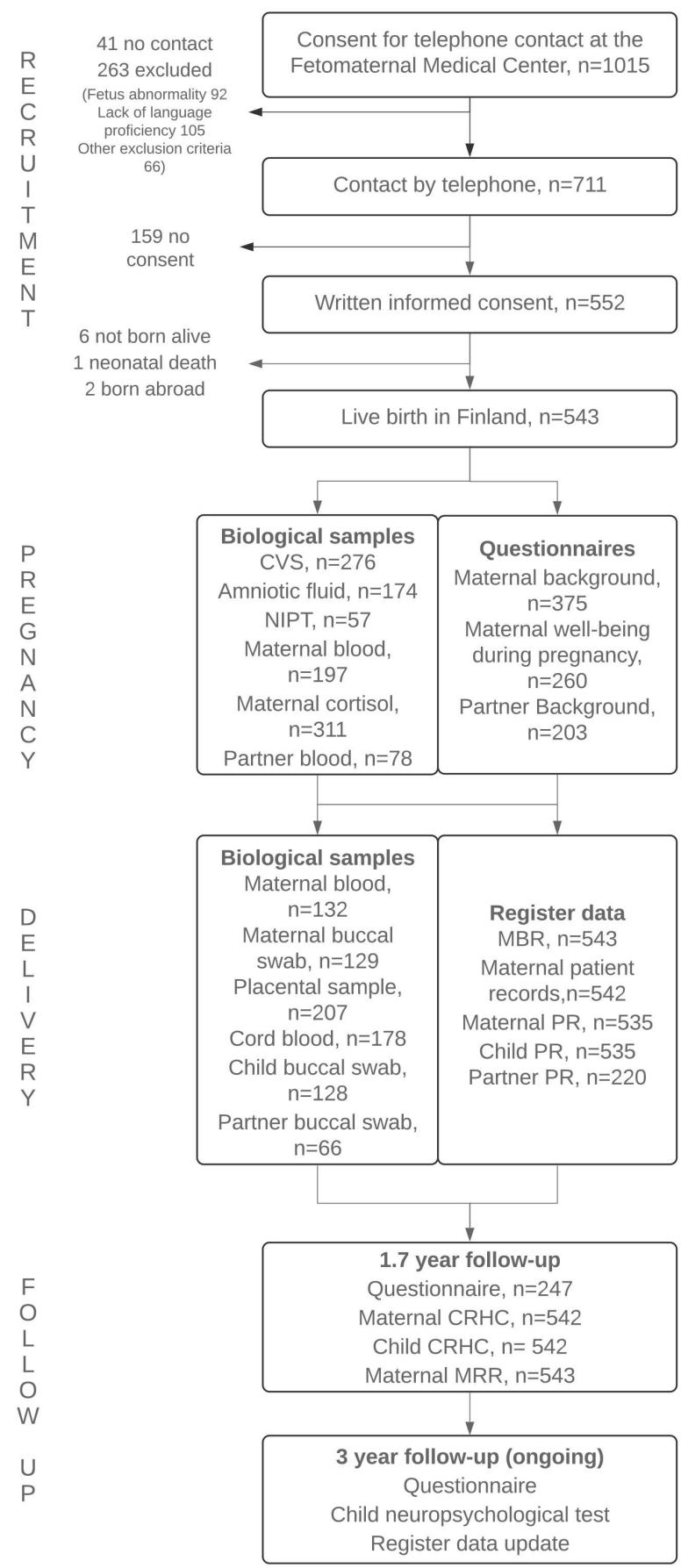

No chromosomal testing arm

mothers who did not undergo CVS, AC nor NIPT

Figure 1 Flow chart of the participants. Maternal cortisol and maternal well-being were measured three times during pregnancy: in early pregnancy ( $<22$ weeks), mid-pregnancy (22-36 weeks) and late pregnancy ( $\geq 36$ weeks). The numbers indicate how many mothers participated at least once. Chorionic villus sampling (CVS), amniotic fluid and non-invasive prenatal testing (NIPT) were collected in early pregnancy. Maternal blood and partner blood were collected once during pregnancy. Majority donated blood in early or mid-pregnancy. AC, amniocentesis; CRHC, Care Register for Health Care; MBR, Medical Birth Register; MRR, Medical Reimbursement Register; PR, Population Register.

offered voluntary fetal chromosomal testing. ${ }^{21}{ }^{23}$ Fetal chromosomal testing including chorionic villus sampling (CVS), amniocentesis (AC), or non-invasive prenatal testing (NIPT), which relies on analysis of cell-free DNA in maternal blood, are used to confirm whether individuals who have a positive screening result have a fetal chromosomal abnormality. ${ }^{24}$ In the Helsinki and Uusimaa Hospital District area, where the current study is set, the Helsinki and Uusimaa Hospital District Fetomaternal Medical Center (FMC) serves the entire population of the area for 
fetal chromosomal testing. ${ }^{21}{ }^{23}$ Until 2015, FMC offered CVS (at $10^{+0}-14^{+6}$ weeks) or AC (week $15^{+0}$ onwards) for chromosomal analysis. In 2015, FMC introduced NIPT, which mostly replaced CVS and AC as the first line of investigation: preliminary chromosomal abnormalities in NIPT were confirmed using CVS or AC. ${ }^{24}$

ITU study has two study arms. For the chromosomal testing arm, we recruited pregnant women who had $a$ positive screening result in the national voluntary prenatal screening programme for trisomy 21 and were thus referred to FMC for fetal chromosomal testing, but who had a negative fetal chromosomal testing result, that is, no fetal chromosomal abnormalities despite an initial positive screening result. This recruitment process had two steps. First, when women visited FMC for chromosomal testing, FMC staff provided information about the ITU study and women gave initial consent to be contacted in case the fetal chromosomal test (CVS/AC/NIPT) suggested no fetal chromosomal abnormality. Second, approximately 1-2 weeks after this FMC visit, a research nurse at FMC checked the chromosomal testing results and contacted the women who tested negative, for final recruitment. Women who had a positive fetal chromosomal testing result were not contacted for final recruitment. Of the 1015 women who provided initial consent to be contacted, 711 had a negative fetal chromosomal testing result and could be reached by phone. Of them, 552 provided final written informed consent to participate in the study. Of these 552 women, 543 delivered a live child in Finland and were thus included in the ITU study chromosomal testing arm.

For the no chromosomal testing arm, we recruited pregnant women who had a negative screening result in the national voluntary prenatal screening programme for trisomy 21 and were thus not referred to the FMC for fetal chromosomal testing. The recruitment process had two steps. First, staff at the clinics conducting the trisomy 21 screening provided initial information about the study and 568 women expressed interest in participating. The research nurse contacted these women within a week of expressed interest: of the 568 women, 558 were reached by phone, and of them, 403 provided written informed consent. Of the 403 women, 401 delivered a live child in Finland and were thus included in the ITU study no chromosomal testing arm.

The recruitment and participation to ITU has been prospective except that in the chromosomal testing arm, the written informed consent allowed research use of the surplus tissue of CVS/amniotic fluid samples taken for fetal chromosomal testing. Study participation is voluntary at all times, and the decision of whether or not to participate in ITU has not affected any treatment decisions.

During the study period, four women participated twice in the chromosomal testing arm, and one woman participated twice in the no chromosomal testing arm. Each pregnancy, and thus each individual mother-child dyad, was given a separate identifier.
At enrolment, we asked all participating mothers for permission to recruit their partner: 480 partners provided written informed consent $(\mathrm{n}=220$ in chromosomal testing arm; $n=260$ in no chromosomal testing arm), which resulted in 480 mother-partner-child triads.

\section{Data collection and measures}

\section{Questionnaire data during pregnancy}

At enrolment, mothers and partners were asked to fill in a background questionnaire, covering education, occupation and income; current and childhood family structure and parental background; reproductive history; current and past health problems; physical activity; diet; and substance and tobacco use.

Mothers completed a detailed well-being questionnaire up to three times during pregnancy: in early pregnancy ( $<22$ weeks), mid-pregnancy (22-36 weeks) and late pregnancy ( $\geq 36$ weeks). This questionnaire covered dietary intake using a food frequency questionnaire ${ }^{25}$ and intake of dietary glycyrrhizin; hyperemesis ${ }^{26}$; sleep quality and quantity $^{27} 28$ and daytime sleepiness ${ }^{29}$; job control ${ }^{30}$ and job demand ${ }^{31}$; perceived symptoms of stress $^{32}$; stressful life events ${ }^{33}$; post-traumatic stress disorder ${ }^{34}$; symptoms of anxiety $^{35}{ }^{36}$ and depression ${ }^{37}$; and positive affect. ${ }^{38}$

\section{Biological tissue samples during pregnancy}

In the chromosomal testing arm, experienced obstetricians performed early-pregnancy CVS and AC sampling based on clinical indication: for the current study, after chromosomal analysis, any surplus tissue was immediately stored at $-80^{\circ} \mathrm{C}$.

Maternal blood samples were collected once during pregnancy. Majority donated blood in early pregnancy (<22 weeks) or mid-pregnancy (22-36 weeks) with a small percentage $(3 \%)$ in late pregnancy ( $\geq 36$ weeks). The samples were collected between 07:00 and 09:00 after a 12-hour fast using antecubital venepuncture: serum, plasma and whole blood samples, and samples in (PAXgene) RNA Tubes were stored at $-80^{\circ} \mathrm{C}$.

Mothers provided a seven-sample set of saliva up to three times during pregnancy: in early pregnancy $(<22$ weeks), mid-pregnancy (22-36 weeks) and late pregnancy ( $\geq 36$ weeks), using polymer rolls (Salivette) to extract saliva when waking up, 15 and 30 min thereafter; at 10:00, noon, 17:00 and when going to sleep ('lights out'). Mothers mailed the salivary samples to the research centre where they were centrifuged and stored at $-20^{\circ} \mathrm{C}$.

\section{Postpartum biological tissue samples}

Immediately after the child was born, midwives extracted cord blood (up to $9 \mathrm{~mL}$ plasma and whole blood stored with ethylenediaminetetraacetic acid).

Within 0-2 hours of delivery, midwives collected buccal swab samples from the mother, child and partner $(2 \times$ buccal swabs per person, Epicentre Biotechnologies Catch-All Sample Collection Swab or Isohelix DNA Buccal Swabs). Neonatal swab samples were taken before 
suckling for the first time. Swab samples were refrigerated for $<24$ hours and then stored at $-80^{\circ} \mathrm{C}$.

Within 0-2hours of delivery, midwives took nine samples from the fetal side of the placental tissue at $2-3 \mathrm{~cm}$ from umbilical cord insertion $(3 \times 3 \times 3 \mathrm{~mm}$ each $)$. These samples were stored in storage reagent meant to protect long-term RNA quality and integrity (Ciagen RNAprotect Tissue Reagent), first at $+5^{\circ} \mathrm{C}$, then at $-20^{\circ} \mathrm{C}$ and $-80^{\circ} \mathrm{C}$ for long-term storage.

Within 24 hours of delivery, trained research staff took additional samples of the placentas. Placentas were refrigerated while waiting for research staff to arrive. Once on-site, research staff measured the placenta, noted any macroscopic abnormalities and collected histological samples (two $1 \mathrm{~cm}$ umbilical cord samples, one $7 \times 7 \mathrm{~cm}$ sample of placental membrane and two $1 \times 1 \times 1 \mathrm{~cm}$ placental tissue samples stored in formalin), and nine placental tissue samples $(3 \times 3 \times 3 \mathrm{~mm})$, which were refrigerated for $<24$ hours and then stored at $-80^{\circ} \mathrm{C}$ for later use. The placental tissue samples were taken from the fetal side, at $2-3 \mathrm{~cm}$ from umbilical cord insertion.

Maternal antecubital venous blood samples were taken $0-2$ days after the delivery and stored at $-80^{\circ} \mathrm{C}$. Partners provided one blood sample during the antenatal or postpartum period: serum, plasma, whole blood and (PAXgene) RNA Tubes were stored at $-80^{\circ} \mathrm{C}$.

\section{Follow-up at the child's age of 1.7 years}

At the child age of 1.7 years (range 1.4-2.2 years), mothers filled in a follow-up questionnaire. The questionnaire included questions related to the mother: maternal education, occupation and income; current family structure; reproductive history; current and past health problems; physical activity; and diet and substance use and smoking history. Furthermore, it included questions on sleep quantity and quality ${ }^{27} 28$ and daytime sleepiness ${ }^{29}$; job control ${ }^{30}$ and job demand ${ }^{31}$; general and parenting stress $^{32}{ }^{39}$; post-traumatic stress disorder ${ }^{34}$; traumatic experiences in childhood ${ }^{40}$; symptoms of anxiety ${ }^{3536}$ and depression ${ }^{37}$; and partner satisfaction. ${ }^{41}$

The questionnaire also included questions related to the child: diet, health problems and illnesses, sleep quantity and quality, ${ }^{42} 43$ temperament and behaviour ${ }^{44}$; social and emotional development, ${ }^{45}$ developmental milestones $^{46}$; and autism-related traits. ${ }^{47}$

\section{Follow-up at the child's age of 3 years}

At the child's target age of 3 years, mothers filled in a questionnaire similar to the one administered during the follow-up at the child's age of 1.7 years, with the following updates: a questionnaire on child gender roles was added ${ }^{48}$ and the questionnaire regarding developmental milestones ${ }^{49}$ was replaced by neuropsychological assessment using the Bayley-III. This test is a widely used and validated instrument for assessing cognitive, expressive language, language comprehension and fine and gross motor abilities. ${ }^{50}$ Testing was performed by trained psychology students and supervised by a clinical paediatric neuropsychologist (EW). Subscale and fullscale scores were calculated using normative data tailored for exact age. ${ }^{51}$

\section{Data from medical records and nationwide registers}

Finland provides free-of-charge maternity care at motherchild clinics, including 10 routine visits between $8-10$ weeks' gestation and 5-12 weeks post partum. ${ }^{52}$ Over $99 \%$ of deliveries occur in public hospitals. ${ }^{53}$ We were able to retrieve medical records from maternity clinics and delivery hospitals for 943 mothers $(99.9 \%$ of participating mothers). Structured data on maternal diseases and complications, medications, progression and mode of delivery, smoking and substance use, reproductive history, infertility treatments, ultrasound and laboratory screening results, weight and blood pressure were extracted by three experienced research nurses.

Finland has detailed, highly reliable nationwide medical registers. ${ }^{54}$ All participating mothers and partners consented to link their data and their child's data with data from these registers, which were obtained with permission from respective registers. The registers have almost $100 \%$ coverage (see figure 1 for number of participants with available data from each register). The register data are currently available until 31 December 2017 and will be regularly updated as the children age.

From the Population Register, ${ }^{55}$ with data available since 1969, we extracted sex, native language, place of residence, marital status, date and place of birth, and date of death or of moving abroad for each mother, her partner and her children.

From the Medical Birth Register, ${ }^{56}{ }^{57}$ which covers all births in Finland since 1987, we extracted detailed information on deliveries, including maternal early-pregnancy body mass index (BMI), maternal and neonatal diagnoses and treatments given during pregnancy and peripartum, reproductive history and fertility treatments, occupation, maternal smoking, prenatal screening results (2017 onwards), delivery mode and birth outcome. We were also able to extract information from the Medical Birth Register on births of 103 ITU mothers themselves, who were born in 1987 or later.

From the Care Register for Health Care with data available on all public and private inpatient hospital stays in Finland since 1969, and on all open-ward hospitalisations and outpatient visits to public specialised healthcare services in Finland since 1998, we extracted recorded diagnoses in the International Statistical Classification of Diseases and Related Health Problems (ICD-10), and comparable codes under previous versions of the ICD as follows: mental and behavioural disorders (category $\mathrm{F}$ in the ICD-10); cardiovascular diseases (category I in the ICD-10); endocrine, nutritional and metabolic diseases (category $\mathrm{E}$ in the ICD-10); diseases of the respiratory system (category J in the ICD-10); and obstetric and perinatal disorders (category O in the ICD-10). Diagnostic data included the ICD code and the date of diagnosis, and supporting treatment-related and injury-related 
information: these data are collected by the Finnish National Institute for Health and Welfare from various healthcare service providers in Finland. ${ }^{58}$

The Medical Reimbursement Register contains information on medication purchases ${ }^{59}$ with data available since 1994. The data also include information from 1964 onwards on a range of diseases entitling the person to medical reimbursements. Finnish residents are covered under the national health insurance programme, which reimburses medication purchases if they fulfil three criteria: first, if the medication was prescribed by a medical doctor/dentist, excluding most over-the-counter products; second, if the medication was purchased in a pharmacy, excluding inpatient medical treatment; and third, if the medication was confirmed as reimbursable by the Pharmaceuticals Pricing Board, ${ }^{60}$ excluding nonapproved medications such as those still undergoing a clinical trial. We retrieved the Anatomical Therapeutic Chemical Classification System (ATC) codes, dates, dosages and indications for all reimbursed medication purchases in the following categories: nervous system medications such as analgesics, antiepileptics, antipsychotic drugs, anxiolytics and antidepressants (ATC category $\mathrm{N}$ ); diabetes and cardiovascular disorders (ATC categories $\mathrm{A} 10$ and $\mathrm{C}$ ); gynaecological/genitourinary medications/contraceptives (ATC category G); endocrine hormone therapies including corticosteroids and thyroid medications (ATC category $\mathrm{H}$ ); systemic antibiotics and viral medication (ATC category J); and respiratory system medications such as asthma and allergy medications (ATC category R).

\section{Genomic, epigenomic and transcriptomic measures}

DNA and RNA were extracted from CVS and delivery placenta, and DNA was extracted from cord blood leucocytes using a bead-based method optimised by tissue type (Chemagic 360, Perkin Elmer). Total CVS were homogenised and split 40\%-60\% for RNA-DNA extraction. Delivery placenta samples preserved in RNAprotect reagent were thawed and equal-sized aliquots were dissected, homogenised and split $40 \%-60 \%$ for RNADNA extraction. Quantification and quality assessments were performed using a TapeStation Automated Electrophoresis System (Agilent) and an Epoch Microplate Spectrophotometer (BioTek, Agilent).

Genotyping was performed on Illumina GSA-24v2-0 A1 arrays according to the manufacturer's guidelines (Illumina, San Diego, California). Quality control was performed in Plink V.1.9 ${ }^{61}$ and R. ${ }^{62}$ DNA was extracted from cord blood, if available, otherwise placental tissue was used. For 24 samples, we extracted DNA from cord blood as well as from placenta. Twenty-three samples presented with perfect matching genotypes from cord blood and placenta, one sample with mismatching genotypes was excluded from the analysis. For 56 samples, we extracted DNA from CVS and cord blood or placenta, all these doublettes presented with consistency rates $\geq 0.99$, indicating that genotyping from CVS tissue worked fine.
Single nucleotide polymorphisms(SNPs) with a call rate below $98 \%$, a minor allele frequency below $1 \%$ or deviation from Hardy-Weinberg equilibrium (HWE) with a $\mathrm{p}$ value $<1 \times 10^{-05}$ were removed from the analysis. SNPs mapping to multiple locations as well as duplicated variants were also removed. Individuals with a genotype call rate below $98 \%$ were also excluded. Duplicated variants were removed. We checked for relatedness using the identify by decent (IBD) matrix. For each related pair, the sibling with higher genotype call rate was included in the analysis, the other sibling was removed. To check for possible population stratification outliers, genotypes were pruned and multidimensional scaling (MDS) analysis on the IBD matrix was performed. Outliers, defined as samples presenting with a position on any of the first ten axes of variation deviating more than 4 SDs from the respective axis' mean, were iteratively removed until no more outliers were detected. Afterwards, individuals presenting with heterozygosity values more than 4 SDs away from the mean heterozygosity were also iteratively removed. Imputation was performed using shapeit $2^{63}$ and impute2. ${ }^{64}$ Chromosomal and base pair positions were updated to the 1000 Genomes Phase 3 reference set, allele strands were flipped where necessary. After imputation, we reran quality control, filtering out SNPs with an info score $<0.6$, a minor allele frequency below $1 \%$ and a deviation fromHWE with a $\mathrm{p}$ value $<1.0 \times 10^{-06}$.

For methylation analyses, $400 \mathrm{ng}$ of DNA from CVS, placenta and cord blood leucocytes was used for bisulfite conversion using the EZ-96 DNA Methylation Kit (Zymo Research). The Infinium Methylation EPIC BeadChip Kit (Illumina) was used to interrogate over 850000 methylation sites quantitatively across the genome at singlenucleotide resolution.

For gene expression analyses, the QuantSeq 3' mRNA-Seq Library Prep Kit FWD by Lexogen (Lexogen, Vienna, Austria) was used to generate libraries from 30 and 50 ng starting material for CVS and delivery placentas, respectively. All libraries were randomised, multiplexed and sequenced on an Illumina HighSeq4000 system at an average depth of 10 million reads per library. Sequencing quality was verified using FastQC ${ }^{65}$ and adapter sequences were trimmed using cutadapt. ${ }^{66}$ For subsequent alignment to the human reference genome GRCh38, we used the STAR aligner ${ }^{67}$ and an adjusted Ensembl gene annotation version 3.0.0 provided by the 10X Genomics Cell Ranger Pipeline tailored to 3 ' sequencing library quantification. We applied featureCounts ${ }^{68}$ to extract gene quantification. For differential expression analysis, analysis tools like DESeq $2^{69}$ and Limma ${ }^{70}$ will be employed. Covariates such as RNA integrity number and library preparation batches as well as surrogate variable analysis ${ }^{71}$ will be taken into account to correct for possible batch effects.

Endocrine, inflammatory and metabolic measures

To date, these analyses have been carried out in maternal salivary and plasma samples during pregnancy. 
From the three consecutive diurnal salivary samples the mothers provided during pregnancy, cortisol has been determined by competitive enzyme immunoassay (ELISA), kit (RE52611, TECAN, IBL, Hamburg, Germany). The standard range was $0.015-3.0 \mu \mathrm{g} / \mathrm{dL}$. The analytical sensitivity (limit of quantitation) is at $0.003 \mathrm{\mu g} /$ $\mathrm{dL}$, the $2 \mathrm{SD}$ functional sensitivity is at $0.005 \mu \mathrm{g} / \mathrm{dL}$ and the mean concentration is $<20 \%$; cross-reactivity of other tested substances $<0.01 \%$; intra-assay $3.2-6.1$; interlot 4.2-17.0.

From the maternal plasma sample taken at early, mid and late pregnancies, a targeted panel of 225 metabolic measures have been quantified by using high-throughput proton nuclear magnetic resonance (NMR) metabolomics platform (Nightingale Health, Helsinki, Finland). These metabolic measures cover multiple metabolic pathways, including lipoprotein lipids and their subclasses, fatty acids, amino acids, ketone bodies and metabolites related to glycolysis and inflammation. Details of the experimentation and applications of the NMR metabolomics platform have been described previously. ${ }^{72}$ This NMR platform has been used in studies of pregnant and non-pregnant populations ${ }^{73-75}$ and shown to correlate with standard clinical chemistry methods for glucose and lipids, ${ }^{73}$ and 37 metabolites in this panel have recently been validated using standard clinical chemistry methods. ${ }^{77}$
Power to detect effects

In general, the ITU study provides us a possibility to detect associations that are small to medium in effect size. In the following calculations, the type I error risk is set to $5 \%$ (alpha $=0.05$ ) and the power is set to $80 \%$ (beta $=0.2$ ). We are able to detect associations between continuous variables which in effect size (Pearson $r$ ) are at least as large as follows: $r=0.09$ in the whole ITU, $r=0.13$ in the subgroup with samples of delivery placenta, $r=0.17$ in the subgroup with CVS and $r=0.21$ in the subgroup with AC. A group difference between the two study arms can be detected in a continuous variable if the assumed difference of means is at least $0.19 \mathrm{SD}$. These calculations assume near complete data availability, which is true at least for the ITU register data. The availability of selfreported data is lower, and for example, in a continuous measure from the 1.7 years of follow-up questionnaire, we can detect a group difference between the study arms if the assumed difference of means is at least 0.24 SD.

\section{FINDINGS TO DATE}

ITU is a newly established data set and no results based on its data have been published to date. However, there are several ongoing projects that address the overarching objectives of the ITU. ITU is also participating in ongoing epigenome-wide association study (EWAS) projects conducted in collaboration with the PACE consortium. ${ }^{15}$ ITU will also contribute data to (genome-wide association

Table 1 Characteristics of the ITU cohort participants, in comparison to all live births in Finland between 2006 and 2017

\begin{tabular}{|c|c|c|c|}
\hline & $\begin{array}{l}\text { ITU } \\
n=944\end{array}$ & $\begin{array}{l}\text { Live births in Finland between } 2006 \\
\text { and } 2017^{*} \\
n=670097\end{array}$ & $\begin{array}{l}\text { Difference } \\
\text { between groups } \dagger\end{array}$ \\
\hline Maternal characteristics & Mean (SD) or $n(\%)$ & Mean (SD) or $\mathrm{n}(\%)$ & $P$ value \\
\hline Age at delivery, years, mean (SD) & $34.8(5.0)$ & $30.4(5.3)$ & $<0.001$ \\
\hline Early-pregnancy BMI, mean (SD) & $24.1(4.3)$ & $24.4(4.9)$ & 0.05 \\
\hline Primiparous, n (\%) & $464(49.2 \%)$ & $278639(41.6 \%)$ & $<0.001$ \\
\hline Hypertension in pregnancy, $\mathrm{n}(\%)$ & $66(7.0 \%)$ & $27732(4.1 \%)$ & $<0.001$ \\
\hline Gestational diabetes, n (\%) & $191(20.2 \%)$ & $76220(11.4 \%)$ & $<0.001$ \\
\hline Smoking during pregnancy, $\mathrm{n}(\%) \ddagger$ & $70(7.4 \%)$ & $98650(14.7 \%)$ & $<0.001$ \\
\hline Antenatal corticosteroid treatment, n (\%) & $32(3.4 \%)$ & $14868(2.2 \%)$ & 0.01 \\
\hline \multicolumn{4}{|l|}{ Child characteristics } \\
\hline
\end{tabular}

$\%$ is the proportion of participants within the data.

$\mathrm{n}$ is the number of participants.

*Birth register data for children born in Finland between 2006 and 2007 are based on previously published data. ${ }^{4}$

†P values were calculated using Pearson $\mathrm{X}^{2}$ tests for categorical variables and using t-tests (equal variances not assumed) for continuous variables. łIn the ITU study, three women had missing data on smoking during pregnancy, otherwise the data presented in this table were complete for all participants. The number of women/children in the Medical Birth Register with available data ranged across items from 656860 to 670097 , as described previously. ${ }^{4}$

BMI, body mass index; ITU, InTraUterine sampling in early pregnancy. 
Table 2 Characteristics of participating mother-child dyads in the two study arms

(1)

\begin{tabular}{|c|c|c|c|}
\hline \multirow[b]{2}{*}{ Maternal characteristics } & & & \multirow{2}{*}{$P$ value } \\
\hline & Mean (SD) or $\mathrm{n}(\%)$ & Mean (SD) or $\mathrm{n}(\%)$ & \\
\hline Early-pregnancy BMI, mean (SD) & $24.6(4.6)$ & $23.5(3.8)$ & $<0.001$ \\
\hline Caesarean section, $\mathrm{n}(\%)$ & $120(22.1 \%)$ & 79 (19.7\%) & 0.37 \\
\hline Hypertension in pregnancy, $\mathrm{n}(\%)$ & $46(8.5 \%)$ & $20(5.0 \%)$ & 0.38 \\
\hline Smoking during pregnancy, $\mathrm{n}(\%) \dagger$ & $56(10.4 \%)$ & $14(3.5 \%)$ & $<0.001$ \\
\hline Antenatal corticosteroid treatment, n (\%) & $23(4.2 \%)$ & $9(2.2 \%)$ & 0.95 \\
\hline \multicolumn{4}{|l|}{ Child characteristics } \\
\hline Sex, girl, n (\%) & $256(47.1 \%)$ & $204(50.9 \%)$ & 0.26 \\
\hline Preterm birth (<37 weeks), n (\%) & $33(6.1 \%)$ & $13(3.2 \%)$ & 0.05 \\
\hline
\end{tabular}

$\%$ is the proportion of women/children within the sample.

$\mathrm{n}$ is the number of participants.

${ }^{*} \mathrm{P}$ values were calculated using Pearson $\mathrm{X}^{2}$ tests for categorical variables and using t-tests (equal variances not assumed) for continuous variables. †Three women in the ITU chromosomal testing arm did not have data on smoking during pregnancy; otherwise, all data presented in this table were complete (available for all participants).

BMI, body mass index; ITU, InTraUterine sampling in early pregnancy.

study (GWAS)) projects conducted in collaboration with the EGG and EAGLE consortia. ${ }^{16}$

Table 1 compares the characteristics of all ITU study participants with characteristics of women and children across all live births in Finland between years 2006 and 2017. ${ }^{4}$ ITU study participants were older, were more often primiparous, more often had hypertensive pregnancy disorders and gestational diabetes, more often had a caesarean section delivery and more often were treated with antenatal corticosteroids and less often smoked during pregnancy than all women giving birth to a live child in Finland between years 2006 and 2017. The groups did not differ in maternal early-pregnancy BMI, child sex, preterm birth or birth weight.

In table 2, we present the findings of the baseline characteristics of the participants in the chromosomal and the no chromosomal testing arms. Women in the chromosomal testing arm were significantly older at delivery, had a higher early-pregnancy BMI, smoked more often during pregnancy, were less often primiparous and more often delivered a preterm baby $(<37$ gestational weeks) than women in the no chromosomal testing arm. Women in these two testing arms did not differ from each other in hypertensive pregnancy disorders or gestational diabetes, mode of delivery or antenatal corticosteroid treatment, and their children did not differ in sex or birth weight.

\section{STRENGTHS AND LIMITATIONS}

A key strength is the unique collection of biological samples from mothers, their partners, placentas and fetuses. Another key strength is that we have collected rare early-pregnancy CVS and amniotic fluid samples, which allow us to study biological mechanisms during the window of early pregnancy. We can also compare early CVS and matching late placental samples, which can be valuable also for biomarker identification. Another strength is the prospective design. Validated data ${ }^{78}$ from patient records and nationwide registers have nearly $100 \%$ coverage. Hence, overall loss to follow-up is minimal, offering an exceptional opportunity to overcome some of the limitations of selective attrition. Moreover, we collected extensive self-reported data using well-validated questionnaires, which cover several key adverse environmental exposures. The ITU study design with its two study arms is also in itself an exposure, and it enables us to compare participants who did versus did not experience a specific stressful life event during pregnancy, that is, positive trisomy 21 screening and subsequent invasive/ non-invasive chromosomal testing.

The ethnic and cultural homogeneity limits generalising the findings to other populations. The representativeness of the ITU sample is limited also within the Finnish population. First, women who underwent fetal chromosomal sampling are over-represented by design, as $52 \%$ of the sample consists of women who underwent fetal chromosomal testing; of the general pregnant population, only $\sim 5 \%$ have an initial positive result in trisomy 21 screening. Second, while over $95 \%$ of the women undergo trisomy 21 screening in early pregnancy, the women who did not participate in this voluntary screening could not be recruited. Third, the ITU recruitment took place in the Helsinki and Uusimaa Hospital District, whose residents 
do not necessarily represent the whole of Finland. Fourth, mothers who were willing to join a voluntary study may have not been representative of the general population. Compared with all pregnancies in Finland between 2006 and 2017, the mean maternal age of ITU participants was higher, they were more often primiparous, smoked less often during pregnancy and the mothers more often received antenatal corticosteroid treatment and experienced hypertensive disorders and gestational diabetes (see table 1 for details). Finally, there is a substantial loss to follow-up in questionnaire and biological data (see figure 1), which further limits representativeness and limits statistical power. The risk of selective recruitment and attrition, and their implications for generalisability, are a key limitation of almost any voluntary follow-up study. Particularly for CVS and AC samples, this limitation is nearly inevitable, because these samples should only be collected in selected populations due to the slightly increased risk of miscarriage. The observed overrepresentation of risk pregnancies could lead to biased effect estimates and limit the generalisability of findings to the general Finnish and other populations. Several statistical techniques, for example, multiple imputation and inverse probability weighting, can help address selection bias: to choose the optimal approach, specific study question and data limitations must be considered. Here, we compared the study population against the general population to advise future statistical approaches and interpretation of findings, and used nationwide register data to address selective attrition.

The questionnaires used in the ITU are standardised and well validated; however, social desirability and response style may inevitably influence responses. Also, smoking and alcohol use during pregnancy are prone to self-report bias and may be under-reported. Comparison of the two study arms is limited by the fact that the recruitment periods did not fully overlap.

\section{Future plans}

Data from the Care Register for Health Care and data from Medical Reimbursement Register on mother, partner and child on ICD-10 diagnoses and medication purchases will be regularly updated. The 3-year follow-up is currently underway. A follow-up is planned in the second grade of primary school at the child's age of 8 years. Apart from data on background characteristics and psychological development, in the future follow-up studies, we plan to collect biological samples to increase the number of mothers and children with biomarker data.

The amniotic fluid samples will be analysed for endocrine, inflammatory and metabolic signatures and RNA extraction and transcriptome analyses in maternal blood samples in early, mid and late pregnancies are underway.

\section{COLLABORATION}

The data sets generated and/or analysed during the current study are not publicly available due to the sensitive patient material. Data requests can be sent to the corresponding author and may be subject to further review by the national register authority and by the ethical committees.

\section{Author affiliations}

${ }^{1}$ Department of Psychology and Logopedics, University of Helsinki, Helsinki, Finland ${ }^{2}$ Department of Child and Adolescent Psychiatry and Psychology, Erasmus MC, Rotterdam, The Netherlands

${ }^{3}$ Department of Translational Research in Psychiatry, Max-Planck Institute of Psychiatry, Munich, Germany

${ }^{4}$ Centre for Cardiovascular Science, Queen's Medical Research Institute, University of Edinburgh, Edinburgh, UK

${ }^{5}$ Singapore Institute for Clinical Sciences, Agency for Science, Technology and Research (A*STAR), Singapore

${ }^{6}$ Department of Obstetrics and Gynaecology, National University of Singapore Yong Loo Lin School of Medicine, Singapore

${ }^{7}$ Department of Obstetrics and Gynecology, University of Helsinki and Helsinki University Hospital, Helsinki, Finland

${ }^{8}$ Medical and Clinical Genetics, Helsinki University Hospital and University of Helsinki, Helsinki, Finland

${ }^{9}$ Helsinki Institute of Life Science, Institute of Molecular Medicine Finland (FIMM), University of Helsinki, Helsinki, Finland

${ }^{10}$ PEDEGO Research Unit, MRC Oulu, Oulu University Hospital and University of Oulu, Oulu, Finland

${ }^{11}$ Public Health Promotion Unit, Finnish Institute for Health and Welfare, Helsinki and Oulu, Finland

Acknowledgements We would like to thank all of the midwives, obstetricians, and other medical staff, laboratory and research personnel, and administrative staff working in hospitals, antenatal and child well-being clinics, municipalities, national registers and other institutions, whose efforts have made this research possible. We would also like to thank a number of research assistants who, during the study period, participated in data collection. Finally, and most importantly, we wish to express our deepest gratitude to all the mothers, partners and children who have participated in the ITU study.

Contributors KR is responsible for the overall content as the guarantor. KR, EK, $\mathrm{JE}, \mathrm{RR}$ and $\mathrm{HL}$ conceived and designed the study. TK wrote the initial draft of the manuscript and analysed and interpreted the data regarding characteristics of the participants. SS played a key role in coordinating the data collection and was a major contributor in writing the manuscript. ML-P has extracted and processed data from various registers and contributed to writing the methods for register data. CC, DC, LD, AT, SR, MR-H and EB processed the endocrine and molecular data and wrote the methods for the genomic, epigenomic and transcriptomic data and diurnal salivary cortisol. EW supervised the neuropsychological testing and revised the manuscript for important intellectual content. EL and SS-K had vital roles in the data collection of ITU and contributed to writing the methods. All authors read and approved the final manuscript.

Funding The ITU study is funded by the Academy of Finland (award numbers: $1284859,12848591,312670,1324596)$ and the Diabetes Research Foundation.

Disclaimer The funder had no role in the design of the ITU study, or conduct of the study, collection, management, analysis, and interpretation of the data, preparation, review, or approval of the manuscript, or decision to submit the manuscript for publication.

Competing interests None declared.

Patient and public involvement Patients and/or the public were not involved in the design, or conduct, or reporting, or dissemination plans of this research.

Patient consent for publication Not required.

Ethics approval This study involves human participants. The ITU research protocol has been approved by the Coordinating Ethics Committee of the Helsinki and Uusimaa Hospital District (approval date: 18 May 2010, reference number: 269/13/03/00/09). The study protocol follows the Helsinki Declaration. Participants gave written informed consent to participate in the study before taking part.

Provenance and peer review Not commissioned; externally peer reviewed.

Data availability statement All data relevant to the study are included in the article or uploaded as supplementary information. 
Open access This is an open access article distributed in accordance with the Creative Commons Attribution Non Commercial (CC BY-NC 4.0) license, which permits others to distribute, remix, adapt, build upon this work non-commercially, and license their derivative works on different terms, provided the original work is properly cited, appropriate credit is given, any changes made indicated, and the use is non-commercial. See: http://creativecommons.org/licenses/by-nc/4.0/.

\section{ORCID iD}

Tuomas Kvist http://orcid.org/0000-0003-3241-8797

\section{REFERENCES}

1 Barker DJP. Maternal nutrition, fetal nutrition, and disease in later life. Nutrition 1997;13:807-13.

2 Razaz N, Villamor E, Muraca GM, et al. Maternal obesity and risk of cardiovascular diseases in offspring: a population-based cohort and sibling-controlled study. Lancet Diabetes Endocrinol 2020;8:572-81.

3 Lahti-Pulkkinen M, Girchenko P, Tuovinen S, et al. Maternal hypertensive pregnancy disorders and mental disorders in children. Hypertension 2020;75:1429-38.

4 Räikkönen K, Gissler M, Kajantie E. Associations between maternal antenatal corticosteroid treatment and mental and behavioral disorders in children. JAMA 2020;323:1924-33.

5 Van den Bergh BRH, van den Heuvel MI, Lahti M, et al. Prenatal developmental origins of behavior and mental health: the influence of maternal stress in pregnancy. Neurosci Biobehav Rev 2020;117:26-64.

6 Robinson R, Lahti-Pulkkinen M, Heinonen K, et al. Fetal programming of neuropsychiatric disorders by maternal pregnancy depression: a systematic mini review. Pediatr Res 2019;85:134-45.

7 Räikkönen K, Seckl JR, Pesonen A-K, et al. Stress, glucocorticoids and liquorice in human pregnancy: programmers of the offspring brain. Stress 2011;14:590-603.

8 Lahti-Pulkkinen M, Cudmore MJ, Haeussner E, et al. Placental morphology is associated with maternal depressive symptoms during pregnancy and toddler psychiatric problems. Sci Rep 2018;8:1-12.

9 Czamara D, Eraslan G, Page CM, et al. Integrated analysis of environmental and genetic influences on cord blood DNA methylation in new-borns. Nat Commun 2019;10:1-18.

10 O'Donnell KJ, Meaney MJ. Fetal origins of mental health: the developmental origins of health and disease hypothesis. Am J Psychiatry 2017;174:319-28.

11 Boyd A, Golding J, Macleod J, et al. Cohort Profile: the 'children of the 90s'--the index offspring of the Avon Longitudinal Study of Parents and Children. Int J Epidemiol 2013;42:111-27.

12 Jaddoe VWV, Mackenbach JP, Moll HA, et al. The generation R study: design and cohort profile. Eur J Epidemiol 2006;21:475-84.

13 Magnus P, Irgens LM, Haug K, et al. Cohort profile: the Norwegian mother and child cohort study (MobA). Int J Epidemiol 2006;35:1146-50.

14 Girchenko P, Lahti M, Tuovinen S, et al. Cohort profile: prediction and prevention of preeclampsia and intrauterine growth restriction (PREDO) study. Int J Epidemiol 2017;46:1380-1.

15 Felix JF, Joubert BR, Baccarelli AA, et al. Cohort profile: pregnancy and childhood epigenetics (PACE) Consortium. Int J Epidemiol 2018;47:22-3.

16 Middeldorp CM, Felix JF, Mahajan A, et al. The early growth genetics (egg) and early genetics and lifecourse epidemiology (Eagle) consortia: design, results and future prospects. Eur J Epidemiol 2019;34:279-300.

17 Girchenko P, Lahti-Pulkkinen M, Heinonen K, et al. Persistently high levels of maternal antenatal inflammation are associated with and mediate the effect of prenatal environmental Adversities on neurodevelopmental delay in the offspring. Biol Psychiatry 2020;87:898-907.

18 Mina TH, Räikkönen K, Riley SC, et al. Maternal distress associates with placental genes regulating fetal glucocorticoid exposure and IGF2: role of obesity and sex. Psychoneuroendocrinology 2015;59:112-22.

19 Räikkönen K, Pesonen A-K, O'Reilly JR, et al. Maternal depressive symptoms during pregnancy, placental expression of genes regulating glucocorticoid and serotonin function and infant regulatory behaviors. Psychol Med 2015;45:3217-26.

20 Reynolds RM, Pesonen A-K, O'Reilly JR, et al. Maternal depressive symptoms throughout pregnancy are associated with increased placental glucocorticoid sensitivity. Psychol Med 2015;45:2023-30.

21 Autti-Rämö I, Koskinen H, Mäkelä M. Raskauden ajan ultraäänitutkimukset JA seerumiseulonnat rakenne- JA kromosomipoikkeavuuksien tunnistamisessa, 2005.
22 Hautala J, Gissler M, Ritvanen A, et al. The implementation of a nationwide anomaly screening programme improves prenatal detection of major cardiac defects: an 11-year national populationbased cohort study. BJOG: Int J Obstet Gy 2019;126:864-73.

23 Saloranta C. Sikiön kromosomipoikkeavuuksien seulonta. Duodecim Terveysportti, 2018. Available: https://www.terveysportti.fi/apps/ltk/ article/ykt00634

24 Chen A, Tenhunen H, Torkki P, et al. Women's choices for invasive or non-invasive testing: influence of gestational age and service delivery. Prenat Diagn 2016;36:1217-24.

25 Erkkola M, Karppinen M, Javanainen J. Validity and reproducibility of a food frequency questionnaire for pregnant Finnish women. $A m \mathrm{~J}$ Epidemiol 2001;154:466-76

26 Lacasse A, Rey E, Ferreira E, et al. Validity of a modified pregnancyunique quantification of emesis and nausea (PUQE) scoring index to assess severity of nausea and vomiting of pregnancy. Am J Obstet Gynecol 2008;198:71.e1-7.

27 Buysse DJ, Reynolds CF, Monk TH, et al. The Pittsburgh sleep quality index: a new instrument for psychiatric practice and research. Psychiatry Res 1989;28:193-213.

28 Partinen M, Gislason T. Basic Nordic sleep questionnaire (BNSQ): a quantitated measure of subjective sleep complaints. J Sleep Res 1995;4:150-5.

29 Johns MW. A new method for measuring daytime sleepiness: the Epworth Sleepiness scale. Sleep 1991;14:540-5.

30 Karasek R, Theorell T. ealthy work: stress, productivity and the reconstruction of working life. New York, NY: Basic Books, 1990.

31 Harris PE. The nurse stress index. Work Stress 1989;3:335-46.

32 Cohen S, To K, Mermelstein R. A global measure of perceived stress author (s): Sheldon Cohen, Tom Kamarck and Robin Mermelstein Source. J Health Soc Behav 1983;24:385-96.

33 Pyykkönen A-J, Räikkönen K, Tuomi T, et al. Stressful life events and the metabolic syndrome: the prevalence, prediction and prevention of diabetes (PPP)-Botnia Study. Diabetes Care 2010;33:378-84.

34 Carlson EB. Psychometric study of a brief screen for PTSD: assessing the impact of multiple traumatic events. Assessment 2001;8:431-41.

35 Spielberger CD, Gorsuch RL, Lushene R. Manual for the state-trait anxiety inventory. Palo Alto, CA: Consulting Psychologists Press, 1983.

36 Beck AT, Steer RA. Manual for the beck anxiety inventory. San Antonio, TX: Psychological Corporation, 1990.

37 Eaton WW, Smith C, Ybarra M. Center for epidemiologic studies depression scale: review and revision (CESD and CESD-R). In: Mahwah NJ, ed. The use of psychological testing for treatment planning and outcomes assessment: instruments for adults. . 3ed edn. US Lawrence Erlbaum Associates Publishers, 2004: Vol. 3. 363-77.

38 Watson D, Clark LA, Tellegen A. Development and validation of brief measures of positive and negative affect: the PANAS scales. J Pers Soc Psychol 1988;54:1063-70.

39 Abidin RR. Parenting stress index: manual. 3rd edn. Odessa, FL: Psychological Assessment Resources, 1995.

40 Bernstein DP, Stein JA, Newcomb MD, et al. Development and validation of a brief screening version of the childhood trauma questionnaire. Child Abuse Negl 2003;27:169-90.

41 Busby DM, Christensen C, Crane DR, et al. A revision of the Dyadic adjustment scale for use with distressed and Nondistressed couples: construct hierarchy and multidimensional scales. J Marital Fam Ther 1995;21:289-308.

42 Sadeh A. A brief screening questionnaire for infant sleep problems: validation and findings for an Internet sample. Pediatrics 2004;113:e570-7.

43 Bruni O, Ottaviano S, Guidetti V, et al. The sleep disturbance scale for children (SDSC) construct ion and validation of an instrument to evaluate sleep disturbances in childhood and adolescence. J Sleep Res 1996;5:251-61.

44 Putnam SP, Gartstein MA, Rothbart MK. Measurement of finegrained aspects of toddler temperament: the early childhood behavior questionnaire. Infant Behav Dev 2006;29:386-401.

45 Carter AS, Briggs-Gowan MJ. Manual for the infant-toddler social \& emotional assessment (ITSEA)-version 2. San Antonio, TX: Psychol Corp, 2006

46 Squires J, Ages BD. Stages questionnaires. In: A parent-completed child monitoring system. 3rd edn (ASQ®-3). Baltimore: Paul H Brookes Publishing Co.,Inc, 2009.

47 Ausderau D, Baranek G. Sensory experiences questionnaire 2013:2770-4.

48 Golombok S, Rust J. The pre-school activities inventory: a standardized assessment of gender role in children. Psychol Assess 1993;5:131-6. 
49 Squires J, Potter L, Bricker D. The ASQ user's guide for the ages \& stages questionnaires: a parent-completed, child-monitoring system. Baltimore, MD: Paul H Brookes Publishing, 1995.

50 Bayley N. Bayley scales of infant and toddler development. Pearson: PsychCorp, 2006.

51 Salo S, Munck P, Uusitalo N. Nancy bayley: bayley scales of infant and toddler development TM - Käsikirja. Helsinki: Psykologien Kustannus Oy, 2008

52 Klemetti R, Hakulinen-Viitanen T. Äitiysneuvolaopas, 2013. Available: http://www.julkari.fi/handle/10024/110521

53 Tom S, Jelena O, Halmesmäki E. Suunnittelematon synnytys sairaalan ulkopuolella Medi-Heli 01:n toiminta-alueella 1.4.200331.12.2005. Finnanest 2007;40:449-52.

54 Heino A, Niinimäki M, Mentula $M$, et al. How reliable are health registers? registration of induced abortions and sterilizations in Finland. Informatics for Health and Social Care 2018;43:310-9.

55 Population Information System. Digital and population data services agency. Available: https://dvv.fi/en/population-information-system [Accessed 22 Oct 2020]

56 Medical Birth Register. National Institute for health and welfare (THL). Available: https://thl.fi/en/web/thlfi-en/statistics/information-onstatistics/register-descriptions/newborns [Accessed 22 Oct 2020].

57 Gissler M. Registration of births and induced abortions in the Nordic countries. Finnish Yearb Popul Res 2010:171-8.

58 National Institute for health and welfare (THL): care register for health care. Available: https://www.thl.fi/en/web/thlfi-en/statistics/ information-on-statistics/register-descriptions/care-register-forhealth-care [Accessed 22 Oct 2020].

59 The social insurance Instituon of Finland (Kela): statistics on reimbursements for medical expenses.

60 The pharmaceuticals pricing board. Available: https://www.hila.fi/en/ [Accessed 22 Oct 2020].

61 Chang CC, Chow CC, Tellier LCAM, et al. Second-Generation PLINK: rising to the challenge of larger and richer datasets. Gigascience 2015;4:1-16.

62 R Core Team. R: a language and environment for statistical computing, 2020. Available: https://www.r-project.org

63 Delaneau O, Marchini J, Zagury J-F. A linear complexity phasing method for thousands of genomes. Nat Methods 2012;9:179-81.
64 IMPUTE2. Available: https://mathgen.stats.ox.ac.uk/impute/impute v2.html [Accessed 23 Oct 2020].

65 Babraham bioinformatics: FastQC. Available: http://www. bioinformatics.babraham.ac.uk/projects/fastqc/ [Accessed 23 Oct 2020].

66 Martin M. Cutadapt removes adapter sequences from highthroughput sequencing reads. EMBnet $J$ 2011:17:10.

67 Dobin A, Davis CA, Schlesinger F, et al. Star: ultrafast universal RNAseq aligner. Bioinformatics 2013;29:15-21.

68 Liao Y, Smyth GK, Shi W. FeatureCounts: an efficient General purpose program for assigning sequence reads to genomic features. Bioinformatics 2014;30:923-30.

69 Love Ml, Huber W, Anders S. Moderated estimation of fold change and dispersion for RNA-Seq data with DESeq2. Genome Biol 2014:15:1-21.

70 Ritchie ME, Phipson B, Wu D, et al. Limma powers differential expression analyses for RNA-sequencing and microarray studies. Nucleic Acids Res 2015;43:e47.

71 Leek JT, Johnsson WE, Parker HS, et al. sva: surrogate variable analysis. R package version 3.36.0 2020.

72 Soininen P, Kangas AJ, Würtz P, et al. Quantitative serum nuclear magnetic resonance metabolomics in cardiovascular epidemiology and genetics. Circ Cardiovasc Genet 2015;8:192-206.

73 Taylor MD, Kutty S. Magnetic resonance imaging computation of intracardiac flow derangements in heart failure dyssynchrony. $A m \mathrm{~J}$ Physiol Heart Circ Physiol 2019;316:H10-12.

74 Wang XL, Liu W, Yu Y-Y, et al. Operando NMR spectroscopic analysis of proton transfer in heterogeneous photocatalytic reactions. Nat Commun 2016;7:11918.

75 Würtz $\mathrm{P}$, Wang Q, Niironen $\mathrm{M}$, et al. Metabolic signatures of birthweight in 18288 adolescents and adults. Int J Epidemiol 2016;45:1539-50.

76 Würtz P, Kangas AJ, Soininen P, et al. Quantitative serum nuclear magnetic resonance metabolomics in large-scale epidemiology: a primer on -Omic technologies. Am J Epidemiol 2017;186:1084-96.

77 Nightingale. Available: https://nightingalehealth.com/ [Accessed 2 Nov 2020].

78 Sund R. Quality of the Finnish hospital discharge register: a systematic review. Scand J Public Health 2012;40:505-15. 